\title{
NEUROFILAMENT LIGHT IN SERUM AND CEREBROSPINAL FLUID IN HIP \\ FRACTURE PATIENTS WITH DELIRIUM
}

\section{RUNNING HEAD:}

Cerebrospinal fluid and serum neurofilament light in delirium

KEY WORDS: Delirium; Blood and CSF biomarker; Dementia; Neuraxonal injury.

\section{AUTHORS}

Nathalie Bodd Halaas, MD; Kaj Blennow, MD, PhD; Ane-Victoria Idland, MD; Torgeir Bruun Wyller, MD, PhD ; Johan Ræder, MD, PhD; Frede Frihagen, MD, PhD; Anne Cathrine Staff, MD, PhD; Henrik Zetterberg, MD, PhD; Leiv Otto Watne, MD, PhD

\section{AFFILIATIONS:}

Nathalie Bodd Halaas

Email: n.b.halaas@psykologi.uio.no.

Oslo Delirium Research Group, Department of Geriatric Medicine, Institute of Clinical Medicine, University of Oslo, Oslo, Norway

Research Group for Lifespan Changes in Brain and Cognition, Department of Psychology, University of Oslo, Oslo, Norway

Kaj Blennow

Email: kaj.blennow@neuro.gu.se

Department of Psychiatry and Neurochemistry, Sahlgrenska Academy, University of Gothenburg, Mölndal, Sweden

Clinical Neurochemistry Laboratory, Sahlgrenska University Hospital, Mölndal, Sweden 
Ane-Victoria Ildand

Email: a.v.idland@medisin.uio.no

Oslo Delirium Research Group, Department of Geriatric Medicine, Institute of Clinical

Medicine, University of Oslo, Oslo, Norway

Institute of Basic Medical Sciences, University of Oslo, Oslo, Norway

Torgeir Bruun Wyller

Email: t.b.wyller@medisin.uio.no

Institute of Clinical Medicine, University of Oslo, Oslo, Norway

Oslo Delirium Research Group, Department of Geriatric Medicine, Oslo University Hospital, Oslo, Norway

Johan Ræder

Email: johan.rader@medisin.uio.no

Department of Anaestesiology, Oslo University Hospital, Oslo, Norway

Institute of Clinical Medicine, University of Oslo, Oslo, Norway

Frede Frihagen

Email: ffrihagen@gmail.com

Division of Orthopaedic Surgery, Oslo University Hospital, Oslo, Norway

Anne Cathrine Staff

Email: uxnnaf@ous-hf.no

University of Oslo, Faculty of Medicine, Institute of Clinical Medicine, Oslo, Norway

Division of Obstetrics and Gynaecology, Oslo University Hospital, Oslo, Norway 
Henrik Zetterberg

Email: henrik.zetterberg@ clinchem.gu.se

Department of Psychiatry and Neurochemistry, Institute of Neuroscience and Physiology, the Sahlgrenska Academy at the University of Gothenburg, Mölndal, Sweden

Clinical Neurochemistry Laboratory, Sahlgrenska University Hospital, Mölndal, Sweden

Department of Neurodegenerative Disease, UCL Institute of Neurology, Queen Square,

London, United Kingdom

UK Dementia Research Institute at UCL, London, United Kingdom

Leiv Otto Watne

Email: 1.o.watne@medisin.uio.no

Oslo Delirium Research Group, Department of Geriatric Medicine, University of Oslo, Oslo, Norway

Institute of Basic Medical Sciences, University of Oslo, Oslo, Norway

\section{CORRESPONDING AUTHOR AND AUTHOR TO ADDRESS FOR REPRINTS:}

Nathalie Bodd Halaas, University of Oslo, Campus Ullevål, Department of Geriatric

Medicine, Postboks 4956 Nydalen, 0424 Oslo, Norway. Telephone: + 47 98640857. E-mail: n.b.halaas@psykologi.uio.no.

\section{CONFLICTS OF INTEREST AND SOURCES OF FUNDING:}

Dr. N.B. Halaas reports no disclosures. Dr. K. Blennow has served as a consultant or at advisory boards for Alzheon, BioArctic, Biogen, Eli Lilly, Fujirebio Europe, IBL International, Merck, Novartis, Pfizer, and Roche Diagnostics, and is a co-founder of Brain 
Biomarker Solutions in Gothenburg AB, a GU Venture-based platform company at the University of Gothenburg. Drs. A-V. Idland, T.B. Wyller, J. Ræder, F. Frihagen and A.CX. Staff report no disclosures. Dr. H. Zetterberg has served at scientific advisory boards for Eli Lilly, Roche Diagnostics and Wave, has received travel support from Teva and is a cofounder of Brain Biomarker Solutions in Gothenburg AB, a GU Ventures-based platform company at the University of Gothenburg. Dr. L.O. Watne reports no disclosures.

The study was funded by the Research Council of Norway, Norwegian National Health Organization, the South-Eastern Norway Regional Health Authorities, the Medical Student Research Program in Norway, the Swedish Research Council, Swedish State Support for Clinical Research and the European Research Council, the Swedish Brain Foundation, the Swedish Alzheimer Foundation. Henrik Zetterberg is a Wallenberg Academy Fellow. Kaj Blennow holds the Torsten Söderberg Professorship in Medicine. The sponsors had no role in the design and conduct of the study; collection, management, analysis, and interpretation of the data; and preparation, review, or approval of the manuscript. 


\section{STRUCTURED ABSTRACT}

Background: Delirium is associated with new-onset dementia, suggesting that delirium pathophysiology involves neuronal injury. Neurofilament light (NFL) is a sensitive biomarker for neuraxonal injury. Methods: NFL was measured in CSF $(n=130)$, preoperative $(n=192)$ and postoperative serum $(n=280)$ in hip fracture patients, and in CSF $(n=123)$ and preoperative serum $(n=134)$ in cognitively normal older adults undergoing elective surgery. Delirium was diagnosed with the confusion assessment method (CAM). Results: Median serum NFL (pg/mL) was elevated in delirium in hip fracture patients (94 vs 54 pre- and 135 vs 92 postoperatively, both $\mathrm{p}<0.001)$. Median CSF NFL tended to be higher in hip fracture patients with delirium (1804 vs 1636, $\mathrm{p}=0.074$ ). Serum and CSF NFL were positively correlated $(\rho=0.56 ; p<0.001)$. Conclusion: Our findings support an association between neuraxonal injury and delirium. The correlation between serum and CSF NFL supports the use of NFL as a blood biomarker in future delirium studies.

\section{INTRODUCTION:}

Delirium is a severe syndrome of acute brain dysfunction characterized by fluctuating awareness, inattention and cognitive symptoms and is associated with serious adverse outcomes and increased healthcare costs [1-3]. Older hospitalized patients are at high risk of developing delirium, and up to $50 \%$ of hip fracture patients are affected $[3,4]$. Delirium seems to increase the risk of new-onset dementia and worsen the long-term trajectory of existing dementia [2,5-8]. The pathophysiological background for this association is unclear as delirium pathophysiology is still poorly understood. 
Emerging literature suggests that delirium may be associated with permanent neuronal damage and/or dysfunction, directly or as a mediator of deleterious insults [2,9]. Studies on biomarkers of brain cell injury have conveyed conflicting results in terms of their association with delirium. Several studies on S100B, a protein expressed primarily in astrocytes in the central nervous system (CNS) [10], have reported increased levels of S100B in delirium patients [11-15]. Others studies, however, did not find similar associations [16-18]. Increased Neuron Specific Enolase (NSE), an enzyme expressed in neurons in the CNS [19], has been reported in delirium patients in three studies [20-22], whereas one study in stroke patients could not replicate these findings [23]. Dissimilar study populations as well as lack of tissue specificity for both S100B and NSE may partly explain the divergent reports. Only a minority of the published studies $[14,15,24]$ has been performed in CSF and, since extracerebral sources of S100B and NSE may influence blood levels, serum changes may not solely reflect upon brain damage [25]. One preliminary study on serum phosphorylated Neurofilament Heavy (pNFH), a structural protein in neurons, found an association between pNFH and delirium severity [26].

Neurofilament light (NFL) is a structural filament protein of the neuronal cytoskeleton and is highly expressed in myelinated large-caliber axons [27-29]. It is a sensitive marker for neuraxonal injury, increasing shortly after and in correspondence to the intensity of brain insult or disease activity [30,31]. In Alzheimer disease, NFL has been shown to increase in relation to amyloid deposition prior to symptom debut in mouse models, and to correlate with lower cognitive scores as well as accelerated rates of cognitive decline in patients [32-34]. NFL in blood and CSF are strongly correlated in patients with different brain disorders, raising the possibility of disease monitoring through blood rather than CSF sampling [35,36]. 
NFL has never been assessed in delirium. Our aim was to examine whether delirium was associated with higher levels of NFL, and assess the correlation between NFL concentrations in CSF and serum.

\section{METHODS}

\section{Cohorts}

Hip fracture cohort

332 patients with proximal femur fractures were recruited to the Oslo Orthogeriatric Trial, a randomized controlled trial evaluating the effect of orthogeriatric care on cognitive function [4,37]. The participants were enrolled at Oslo University Hospital from September 2009 to January 2012. Patients were not included if they were terminally ill or if the fracture resulted from a high-energy trauma. The intervention had no effect on delirium incidence [4] and all participants were pooled in the present study.

Participants were evaluated daily for delirium preoperatively and until the fifth postoperative day (all) or discharge (patients with delirium), using the Confusion Assessment Method (CAM). The study physician or nurse scored CAM based on a 10- to 30-minute interview with participants combined with information from relatives, nurses and hospital records. Depending on time of onset and criteria fulfillment, delirium was categorized as preoperative, incident (i.e. no delirium preoperatively, but developed delirium during the hospital stay), subsyndromal (SSD, defined as at least two positive CAM features [pre- or postoperatively] but never fulfilled criteria for full delirium) and no delirium. When dichotomizing on delirium status, patients with no delirium included patients without any delirium symptoms or SSD and patients with delirium included patients with preoperative or incident delirium. Two specialists (one geriatrician and one 'old age psychiatrist') independently evaluated each 
participant's fulfillment of the ICD-10 criteria for dementia prior to the fracture (i.e. consensus diagnosis for dementia), based on all existing data (except delirium status during admission), including the Informant Questionnaire on Cognitive decline in the Elderly (IQCODE), hospital records (including previous diagnosis of dementia) and data from the follow up controls 4 and 12 months after surgery. The inter-rater consensus agreement upon the dementia diagnosis was reasonable (kappa 0.87). In order to reach a consensus diagnosis, disagreements were resolved through discussion.

Cognitively healthy control group 172 patients undergoing elective gynecological, orthopedic or urological surgery in spinal anesthesia, aged 65 years or older the year of inclusion, were recruited to the COGNORMstudy from 2012-2013 at Oslo University Hospital and Diakonhjemmet Hospital, Oslo, as previously described [38]. Participants were evaluated with a multidomain battery of cognitive tests prior to surgery. Dementia, previous stroke with sequelae, Parkinson's disease and other acknowledged or suspected brain disease likely to influence cognition were exclusion criteria. Furthermore, we excluded patients without CSF or serum samples ( $n=6)$, suspected undiagnosed dementia with referral to a memory clinic by a geriatrician (based on test scores and clinical data) at any time point during five years of follow-up ( $\mathrm{n}=15)$ or MMSE <28 at baseline $(n=16)$. All patients were free from delirium at the time of surgery. Delirium was not assessed postoperatively.

\section{Sampling and biochemical analyses}

CSF was collected in propylene tubes in conjunction with anesthesia prior to administration of the anesthetic agent in both cohorts. Blood was collected by venous puncture preoperatively in both cohorts as well as postoperatively in hip fracture patients. For logistical reasons, 
samples were taken at irregular time intervals pre- and postoperatively and all types of sampling were not obtainable for all patients. Serum and CSF were centrifuged, aliquoted and stored at $-80^{\circ}$ C. Samples were thawed, aliquoted and sent on dry ice (hip fracture patients) or sent directly on dry ice (cognitively healthy controls) for analyses at the Clinical Neurochemistry Laboratory at Sahlgrenska University Hospital (Mölndal, Sweden). CSF NFL concentrations were measured using a commercial ELISA (UmanDiagnostics, Umeå, Sweden) [39]. Serum NFL levels (sNFL) were analysed using a homebrew Single molecule array method [40].

\section{Statistical methods}

Longitudinal changes in serum NFL (sNFL) were calculated as the difference in sNFL pre-to postoperatively. Data from CSF and serum were not normally distributed. Transformation improved fit to the normal distribution only for preoperative serum and did not change the results; therefore non-transformed data are reported. Continuous variables were analyzed using Mann-Whitney $U$ test (unpaired group comparisons), Kruskal-Wallis (unpaired subgroup analyses) and Wilcoxon signed-rank test (paired comparisons). Categorical variables were analyzed using Chi Square statistics. Correlations were calculated with Spearman's rho $(\rho)$.

Since dementia is a major risk factor for delirium [40-41], we also carried out the analyses stratified on dementia status. In order to adjust for confounding factors, linear regression analyses were performed using delirium and age as independent variables. To achieve normal distribution of residuals, sNFL/ CSF NFL and sNFL change were transformed with the natural logarithm in the regression analyses. The linear regression coefficient $\beta$ is reported in logarithmic units of NFL. 
We further wanted to explore whether time of symptom debut had any impact on NFL levels at time of sampling. Thus analyses on delirium status at the time of CSF sampling (preoperative versus incident versus subsyndromal (SSD) versus no delirium) were performed posthoc. A recent study indicates that SSD may be symptomatologically closer to full-blown delirium than to no delirium [42], and all analyses were therefore repeated excluding patients with SSD. Furthermore potentially delirium-associated release of NFL likely begins prior to substrate sampling in patients with preoperative delirium. Measuring change of NFL pre-to postoperatively in this subgroup may therefore underestimate the release of NFL significantly. Hence, the analyses on change were repeated and compared between incident delirium and no delirium, excluding patients with preoperative delirium $(n=41)$.

All statistical analyses were performed using SPSS Statistics version 25 (IBM, Armonk, NY). Graphs were designed using GraphPad Prism (https://www.graphpad.com/scientificsoftware/prism/).

\section{Standard Protocol Approvals, Registrations, and Patient Consents}

The study was conducted in accordance with the Declaration of Helsinki. The data and serum/ CSF samples were collected after informed consent from the patient and/or proxy (if patients were unable to consent due to cognitive impairment), as approved by the Regional Committee for Medical and Health Research Ethics (South-East Norway; REK 2009/450 and REK 2011/2052).

\section{Data availability statement}

Data are available on request to the authors. 


\section{RESULTS}

CSF NFL was analyzed in 130 hip fracture patients and 123 cognitively healthy controls. In serum, preoperative levels of NFL were analyzed in 192 hip fracture patients and 134 controls, and postoperative levels were analyzed in 280 hip fracture patients. Changes in sNFL pre- to postoperatively were calculated in 162 hip fracture patients (Figure 1).

\section{Hip fracture patients}

Patients with delirium were slightly older, had a higher median IQCODE score and higher percentage of dementia than delirium-free hip fracture patients (Table 1).

Neurofilament in serum (sNFL)

Pre - and postoperative sNFL

Patients with delirium had significantly higher pre- and postoperative sNFL compared to patients without delirium (median 94 vs. 54 pg/mL preoperatively, p<0.001 and 135 vs. 92 $\mathrm{pg} / \mathrm{mL}$ postoperatively, $\mathrm{p}<0.001$ ) (Figure 2). The associations were also found in regression analysis adjusting for age (preoperative, $\beta=0.37, \mathrm{p}=0.001$ and postoperative, $\beta=0.27$, $\mathrm{p}=0.001$ ) (Supplementary table 1 ). sNFL seemed related to the time of onset of symptoms, with highest NFL values in preoperative serum measured in patients with preoperative delirium and in postoperative serum measured in patients with incident delirium (Figure 3AB).

Patients with dementia had significantly higher levels of NFL pre- and postoperatively than patients without dementia (median 98 vs 53 pg/mL preoperatively and 130 vs 93 pg/mL postoperatively, both $\mathrm{p}<0.001$ ) (Table 2). When stratifying for dementia, NFL remained significantly higher in delirium patients in the dementia-free stratum preoperatively (median 88 vs 46 pg/mL, p=0.001) and postoperatively (median 136 vs 80 pg/mL, p<0.001). This 
association was upheld in a regression analysis with age, $\beta=0.43, \mathrm{p}=0.014$ (preoperatively) and $\beta=0.33, p=0.009$ (postoperatively) (Supplementary table 1). There was no difference between patients with and without delirium in the stratum with dementia (Table 2).

Longitudinal changes in SNFL

sNFL was increased significantly pre- to postoperatively in all groups independently of delirium and dementia status ( $45 \%$ on average in the whole hip fracture cohort). The magnitude of this alteration did not differ significantly between patients with delirium and the remaining hip fracture population ( $\mathrm{p}=0.080$, Mann Whitney- $U$ ). When excluding patients with preoperative delirium, the increase was significantly higher in patients with delirium than in patients without delirium both in the overall hip fracture group (median 75 vs $27, \mathrm{p}=0.006$ ) and in patients with dementia (median 94 vs 24, p<0.001) (Figure 4). In a linear regression adjusting for age, the associations remained significant $(\beta=0.57, p=0.020$ in overall population; $\beta=0.93 . p=0.009$ in the dementia stratum) (Supplementary table 1 ). No difference was found in the dementia-free stratum $(\mathrm{p}=0.93$, Table 2$)$.

\section{Neurofilament in CSF}

Patients with delirium $(n=71)$ tended to have higher median levels of NFL than patients without delirium ( $\mathrm{n}=59), 1804$ vs $1636 \mathrm{pg} / \mathrm{mL}, \mathrm{p}=0.074$ (Table 1). In pairwise subgroup comparisons using Mann Whitney $U$-test, median NFL was higher in patients with preoperative $(n=44)$ than no delirium $(n=37), 1845 \mathrm{vs} 1267 \mathrm{pg} / \mathrm{mL}, \mathrm{p}=0.011$ (Figure 3C). Surprisingly, NFL was numerically highest in patients with SSD ( $\mathrm{n}=20$, median $2044 \mathrm{pg} / \mathrm{mL})$ (Figure 3D). When patients with SSD were removed from the analyses, median NFL was significantly higher in delirium compared to no delirium ( $\mathrm{n}=37), 1804$ vs $1267 \mathrm{pg} / \mathrm{mL}$, 
$\mathrm{p}=0.018$. However, after adjustment for age, the association between NFL and delirium was no longer statistically significant $(\beta=0.21, \mathrm{p}=0.16)$ (Table 1$)$.

Patients with dementia ( $\mathrm{n}=65)$ had significantly higher median levels of NFL than patients without dementia $(n=65) ; 1961$ vs $1526 \mathrm{pg} / \mathrm{mL}, \mathrm{p}=0.025$. No significant difference in CSF NFL concentrations was found between patients with and without delirium in either dementia strata (Table 2).

\section{Cognitively healthy controls}

Patients in the control group were younger than the hip fracture patients ( 71 vs 85 years, $\mathrm{p}<0.001)$ with a more equal gender distribution (Table 1).

NFL was significantly higher in the hip fracture patients than in cognitively normal controls, both in preoperative serum (153\% higher) and CSF (67\% higher) (Table 1). A positive correlation between age and NFL in both serum and CSF ( $\rho=0.49$ and $\rho=0.47, p<0.001)$ was shown.

\section{Correlations in blood and cerebrospinal fluid}

NFL concentrations in serum and CSF correlated positively in both the hip fracture patient group (preoperative serum, $\rho=0.56$ and $p<0.001$ ) as well as in the cognitively healthy controls $(\rho=0.69, p<0.001)$ (Figure 5).

\section{DISCUSSION}

In hip fracture patients, pre- and postoperative sNFL were significantly higher in delirium, also after adjusting for age. After stratifying for dementia, similar findings were found in the dementia-free stratum. Furthermore, CSF NFL was higher in delirium, but this difference did, 
however, not reach statistical significance. The association between elevated NFL and delirium supports the hypothesis that delirium may be connected with neuronal injury $[2,9]$. Increased NFL in delirium patients may reflect brain vulnerability due to reduced neuronal capacity with increased risk of delirium, or that specific pathophysiological mechanisms leading to delirium result in cell injury at the time of syndrome onset. An exaggerated reaction to a generalized stress response following a hip fracture may possibly lead to cell injury and NFL release in a vulnerable brain [43]. Recent studies have suggested that delirium in dementia patients may multiplicatively contribute to the pathological processes in dementia by alternative unknown pathways causing neuronal death $[6,44]$.

Repeated measurements can yield increased insight into the relationship between delirium and cell injury. A larger increase in sNFL in patients with delirium compared to patients without delirium between two time points would strengthen the direct association between delirium and cell injury.

In all patients and subgroups, serum NFL increased significantly from the preoperative to the postoperative phase. In elective surgery patients undergoing general anesthesia, NFL in serum has been found to increase to a maximum 48 hours postoperatively [45]. Damage to peripheral nerves during surgery, noxious effects of anesthesia [46] and systemic physiological reactions could be possible underlying mechanisms. Although patients included in this study were subjected to spinal anesthesia, toxic and/or hemodynamic effects of anesthesia cannot be excluded.

Patients who were delirium-free initially, but developed delirium after surgery (i.e. incident delirium) had the highest increase of NFL, followed by patients with preoperative delirium, SSD and finally no delirium (Figure 3D). Furthermore, highest preoperative levels of sNFL (and CSF NFL) were measured in patients with preoperative delirium and highest 
postoperative levels in patients developing delirium postoperatively (Figure 3 A-C).

Hypothetically, this pattern of sNFL changes in subgroups could illustrate a process whereby cell injury occurs prior to development of delirium symptoms, followed by a NFL peak during the episode and shortly thereafter, a gradual decrease or stabilization of NFL release. A recent study on traumatic brain injury suggests that outflow of NFL may be bimodal [47]. The bimodality may be due to initial outflow from acutely damaged neurons, followed by a decrease and a secondary peak due to secondary axonal degeneration [48]. In posthoc analyses we therefore assumed that potential delirium-associated NFL release might already be in progression in patients with delirium preoperatively and that including these patients could presumably underestimate NFL changes. We thus compared sNFL-change in incident delirium to the corresponding change in delirium-free patients. The NFL increase was significantly larger in patients who developed delirium subsequent to the first serum sampling, compared to patients without delirium. In the dementia stratum this difference was highly significant, also after age adjustment. This indicates that NFL release and neuraxonal injury may be directly involved in delirium pathophysiology in patients with dementia, accounting for the increased risk of dementia progression. No difference was found in dementia-free patients, suggesting that different pathophysiological trajectories, comprising white matter change to a lesser degree, may be involved in development of new-onset dementia after delirium, or that neuronal death in delirium in the neurodegenerative brain may be more extensive and hence detectable $[43,49]$.

Robust correlations were found between CSF and serum NFL in the overall group, in delirium and in dementia patients, in line with findings in other brain disorders [35,36]. Spinal tapping is an invasive procedure that is clinically required only in a minor subset of delirium patients 
[50]. Robust validity of NFL measurements in blood would greatly facilitate use of NFL as a biomarker in research on delirium pathophysiology.

Strengths of our study comprise daily bedside assessment of delirium based on validated instruments. Furthermore the large number of included patients allowed for subgroups analyses. We included both patients with and without dementia as well as a cohort of healthy controls in order to present a wide neurodegenerative specter. Moreover serum was sampled repeatedly and paired with preoperatively CSF sampling, and analyses were performed in an expert laboratory.

Our study was faced with certain limitations. Firstly, NFL levels in the elderly hip fracture patients were significantly higher than in elective controls. This highlights the importance of using matched controls also in delirium research. Comorbidities, such as dementia [51], and greater age $[38,52]$ in an overall frailer hip fracture population are associated with increased levels of NFL in blood and CSF, as we confirmed. A stronger correlation between NFL in CSF and blood in the elective patients compared to the hip fracture patients further suggests that extracerebral sources of NFL are of greater importance in measured sNFL in the hip fracture population.

Secondly, due to logistical reasons, time between blood samplings varied and CSF and serum samples were not available from all patients at all time points. Bimodality of NFL release [47] could implicate that timing of serum samplings may have been inadequate to demonstrate maximal increases and change in serum NFL, reducing the sensitivity of our measurements.

Thirdly, we did not have information regarding dementia etiology. 
This is the first study to show that delirium is associated with increased NFL in CSF and serum, suggesting an association between delirium and neuraxonal injury. Larger sNFL increases demonstrated in incident delirium suggest that mechanisms directly related to delirium pathophysiology may be responsible. Robust correlations found between CSF and serum NFL indicate that future analyses of NFL in delirium patients may be performed in serum, obsoleting cerebrospinal fluid sampling.

\section{ACKNOWLEDGMENTS}

We would like to thank the patients and staff at the Orthopedic Department, the Gynecological Department, the Urology Department and the Anesthesiological Department at Oslo University Hospital and the Surgical Department and the Anesthesiological Department at Diakonhjemmet Hospital in Oslo. We also thank research nurses Elisabeth Fragaat and Tone Fredriksen for help in data collection and laboratory technicians at the Clinical Neurochemistry Laboratory, Sahlgrenska University Hospital, for skilful technical analyses. 


\section{REFERENCES}

1. Leslie DL, Marcantonio ER, Zhang Y, Leo-Summers L, Inouye SK. One-year health care costs associated with delirium in the elderly population. Archives of internal medicine.

2008;168:27-32.

2. Fong TG, Davis D, Growdon ME, Albuquerque A, Inouye SK. The interface between delirium and dementia in elderly adults. The Lancet Neurology. 2015;14:823-832.

3. Inouye SK, Westendorp RG, Saczynski JS. Delirium in elderly people. Lancet (London, England). 2014;383:911-922.

4. Watne LO, Torbergsen AC, Conroy S, Engedal K, Frihagen F, Hjorthaug GA, Juliebo V, Raeder J, Saltvedt I, Skovlund E, Wyller TB. The effect of a pre- and postoperative orthogeriatric service on cognitive function in patients with hip fracture: randomized controlled trial (Oslo Orthogeriatric Trial). BMC medicine. 2014;12:63.

5. Cunningham C, Campion S, Lunnon K, Murray CL, Woods JF, Deacon RM, Rawlins JN, Perry VH. Systemic inflammation induces acute behavioral and cognitive changes and accelerates neurodegenerative disease. Biological psychiatry. 2009;65:304-312.

6. Davis DHJ, Terrera GM, Keage H, Rahkonen T, Oinas M, Matthews FE, Cunningham C, Polvikoski T, Sulkava R, MacLullich AMJ, Brayne C. Delirium is a strong risk factor for dementia in the oldest-old: a population-based cohort study. Brain. 2012;135:2809-2816.

7. Krogseth M, Watne LO, Juliebo V, Skovlund E, Engedal K, Frihagen F, Wyller TB. Delirium is a risk factor for further cognitive decline in cognitively impaired hip fracture patients. Arch Gerontol Geriatr. 2016;64:38-44.

8. Krogseth M, Wyller TB, Engedal K, Juliebo V. Delirium is an important predictor of incident dementia among elderly hip fracture patients. Dementia and geriatric cognitive disorders. 2011;31:63-70.

9. Maclullich AM, Anand A, Davis DH, Jackson T, Barugh AJ, Hall RJ, Ferguson KJ, Meagher DJ, Cunningham $C$. New horizons in the pathogenesis, assessment and management of delirium. Age Ageing. 2013;42:667-674.

10. Michetti F, Corvino V, Geloso MC, Lattanzi W, Bernardini C, Serpero L, Gazzolo D. The S100B protein in biological fluids: more than a lifelong biomarker of brain distress. Journal of neurochemistry. 2012;120:644-659.

11. van Munster BC, Bisschop PH, Zwinderman AH, Korevaar JC, Endert E, Wiersinga WJ, van Oosten HE, Goslings JC, de Rooij SE. Cortisol, interleukins and S100B in delirium in the elderly. Brain and cognition. 2010;74:18-23.

12. van Munster BC, Korevaar JC, Korse CM, Bonfrer JM, Zwinderman AH, de Rooij SE. Serum S100B in elderly patients with and without delirium. International journal of geriatric psychiatry. 2010;25:234-239.

13. van Munster BC, Korse CM, de Rooij SE, Bonfrer JM, Zwinderman AH, Korevaar JC. Markers of cerebral damage during delirium in elderly patients with hip fracture. BMC neurology. 2009;9:21.

14. Hov KR, Bolstad N, Idland AV, Zetterberg H, Blennow K, Chaudhry FA, Frihagen F, Raeder J, Wyller TB, Watne LO. Cerebrospinal Fluid S100B and Alzheimer's Disease Biomarkers in Hip Fracture Patients with Delirium. Dementia and Geriatric Cognitive Disorders Extra. 2017;7:374-385.

15. Hall RJ, Ferguson KJ, Andrews M, Green AJ, White TO, Armstrong IR, MacLullich AM. Delirium and cerebrospinal fluid S100B in hip fracture patients: a preliminary study. The American journal of geriatric psychiatry : official journal of the American Association for Geriatric Psychiatry. 2013;21:1239-1243.

16. Beishuizen SJ, Scholtens RM, van Munster BC, de Rooij SE. Unraveling the Relationship Between Delirium, Brain Damage, and Subsequent Cognitive Decline in a Cohort of Individuals Undergoing Surgery for Hip Fracture. Journal of the American Geriatrics Society. 2017;65:130-136. 
17. Beishuizen SJ, Scholtens RM, Vellekoop AE, Vrouenraets BC, Westhoff $D$, van de Beek $D$, de Rooij SE, van Munster BC. Timing Is Critical in Determining the Association Between Delirium and S100 Calcium-Binding Protein B. Journal of the American Geriatrics Society.

2015;63:2212-2214.

18. Jorge-Ripper C, Aleman MR, Ros R, Aguilera S, Gonzalez-Reimers E, Espelosin E, Santolaria F. Prognostic value of acute delirium recovery in older adults. Geriatrics \& gerontology international. 2017;17:1161-1167.

19. Haque A, Polcyn R, Matzelle D, Banik NL. New Insights into the Role of Neuron-Specific Enolase in Neuro-Inflammation, Neurodegeneration, and Neuroprotection. Brain sciences. 2018;8.

20. Grandi C, Tomasi CD, Fernandes K, Stertz L, Kapczinski F, Quevedo J, Dal-Pizzol F, Ritter C. Brain-derived neurotrophic factor and neuron-specific enolase, but not S100beta, levels are associated to the occurrence of delirium in intensive care unit patients. Journal of critical care. 2011;26:133-137.

21. Anderson BJ, Chesley CF, Theodore M, Christie C, Tino R, Wysoczanski A, Ramphal K, Oyster M, Kalman L, Porteous MK, Bermudez CA, Cantu E, Kolson DL, Christie JD, Diamond JM. Incidence, risk factors, and clinical implications of post-operative delirium in lung transplant recipients. The Journal of heart and lung transplantation : the official publication of the International Society for Heart Transplantation. 2018.

22. Anderson BJ, Reilly JP, Shashaty MGS, Palakshappa JA, Wysoczanski A, Dunn TG, Kazi A, Tommasini A, Mikkelsen ME, Schweickert WD, Kolson DL, Christie JD, Meyer NJ. Admission plasma levels of the neuronal injury marker neuron-specific enolase are associated with mortality and delirium in sepsis. Journal of critical care. 2016;36:18-23.

23. Kozak HH, Uguz F, Kilinc I, Uca AU, Serhat Tokgoz O, Akpinar Z, Ozer N. Delirium in patients with acute ischemic stroke admitted to the non-intensive stroke unit: Incidence and association between clinical features and inflammatory markers. Neurologia i neurochirurgia polska. 2017;51:38-44.

24. Beishuizen SJ, Scholtens RM, Vellekoop AE, Vrouenraets BC, Westhoff $D$, van de Beek $D$, de Rooij SE, van Munster BC. Timing Is Critical in Determining the Association Between Delirium and S100 Calcium-Binding Protein B. Journal of the American Geriatrics Society. 2015;63:2212-2214.

25. Savola O, Pyhtinen J, Leino TK, Siitonen S, Niemela O, Hillbom M. Effects of head and extracranial injuries on serum protein $\mathrm{S} 100 \mathrm{~B}$ levels in trauma patients. The Journal of trauma. 2004;56:1229-1234; discussion 1234.

26. Inoue R, Sumitani M, Ogata T, Chikuda H, Matsubara T, Kato S, Shimojo N, Uchida K, Yamada $Y$. Direct evidence of central nervous system axonal damage in patients with postoperative delirium: A preliminary study of $\mathrm{pNF}-\mathrm{H}$ as a promising serum biomarker. Neuroscience letters. 2017;653:39-44.

27. Lee MK, Xu Z, Wong PC, Cleveland DW. Neurofilaments are obligate heteropolymers in vivo. The Journal of cell biology. 1993;122:1337-1350.

28. Zetterberg H. Neurofilament Light: A Dynamic Cross-Disease Fluid Biomarker for Neurodegeneration. Neuron. 2016;91:1-3.

29. Friede RL, Miyaghishi T, Hu KH. Axon calibre, neurofilaments, microtubules, sheath thickness and cholesterol in cat optic nerve fibres. Journal of anatomy. 1971;108:365-373.

30. Zetterberg H, Hietala MA, Jonsson M, Andreasen N, Styrud E, Karlsson I, Edman A, Popa C, Rasulzada A, Wahlund LO, Mehta PD, Rosengren L, Blennow K, Wallin A. Neurochemical aftermath of amateur boxing. Archives of neurology. 2006;63:1277-1280.

31. Teunissen CE, Khalil M. Neurofilaments as biomarkers in multiple sclerosis. Multiple sclerosis (Houndmills, Basingstoke, England). 2012;18:552-556.

32. Bacioglu M, Maia LF, Preische O, Schelle J, Apel A, Kaeser SA, Schweighauser M, Eninger T, Lambert M, Pilotto A, Shimshek DR, Neumann U, Kahle PJ, Staufenbiel M, Neumann M, Maetzler W, Kuhle J, Jucker M. Neurofilament Light Chain in Blood and CSF as Marker of 
Disease Progression in Mouse Models and in Neurodegenerative Diseases. Neuron. 2016;91:494-496.

33. Skillback T, Farahmand B, Bartlett JW, Rosen C, Mattsson N, Nagga K, Kilander L, Religa D, Wimo A, Winblad B, Rosengren L, Schott JM, Blennow K, Eriksdotter M, Zetterberg H. CSF neurofilament light differs in neurodegenerative diseases and predicts severity and survival. Neurology. 2014;83:1945-1953.

34. Zetterberg H, Skillback T, Mattsson N, Trojanowski JQ, Portelius E, Shaw LM, Weiner MW, Blennow K. Association of Cerebrospinal Fluid Neurofilament Light Concentration With Alzheimer Disease Progression. JAMA neurology. 2016;73:60-67.

35. Novakova L, Zetterberg $H$, Sundstrom $P$, Axelsson $M$, Khademi $M$, Gunnarsson $M$, Malmestrom C, Svenningsson A, Olsson T, Piehl F, Blennow K, Lycke J. Monitoring disease activity in multiple sclerosis using serum neurofilament light protein. Neurology. 2017;89:2230-2237.

36. Zetterberg $\mathrm{H}$. Fluid biomarkers for microglial activation and axonal injury in multiple sclerosis. Acta Neurol Scand. 2017;136:15-17.

37. Wyller TB, Watne LO, Torbergsen A, Engedal K, Frihagen F, Juliebo V, Saltvedt I, Skovlund E, Raeder J, Conroy $S$. The effect of a pre- and post-operative orthogeriatric service on cognitive function in patients with hip fracture. The protocol of the Oslo Orthogeriatrics Trial. BMC geriatrics. 2012;12:36.

38. Idland AV, Sala-Llonch R, Borza T, Watne LO, Wyller TB, Braekhus A, Zetterberg H, Blennow K, Walhovd KB, Fjell AM. CSF neurofilament light levels predict hippocampal atrophy in cognitively healthy older adults. Neurobiology of aging. 2016;49:138-144.

39. Rosengren LE, Karlsson JE, Karlsson JO, Persson LI, Wikkelso C. Patients with amyotrophic lateral sclerosis and other neurodegenerative diseases have increased levels of neurofilament protein in CSF. Journal of neurochemistry. 1996;67:2013-2018.

40. Kuhle J, Barro C, Andreasson U, Derfuss T, Lindberg R, Sandelius A, Liman V, Norgren N, Blennow $\mathrm{K}$, Zetterberg $\mathrm{H}$. Comparison of three analytical platforms for quantification of the neurofilament light chain in blood samples: ELISA, electrochemiluminescence immunoassay and Simoa. Clin Chem Lab Med. 2016;54:1655-1661.

41. Gual N, Morandi A, Perez LM, Britez L, Burbano P, Man F, Inzitari M. Risk Factors and Outcomes of Delirium in Older Patients Admitted to Postacute Care with and without Dementia. Dementia and geriatric cognitive disorders. 2018;45:121-129.

42. Boettger S, Nunez DG, Meyer R, Richter A, Schubert M, Jenewein J. Subsyndromal delirium in the intensive care setting: Phenomenological characteristics and discrimination of subsyndromal delirium versus no and full-syndromal delirium. Palliative $\&$ supportive care. 2018;16:3-13.

43. MacLullich AMJ, Ferguson KJ, Miller T, de Rooij SEJA, Cunningham C. Unravelling the pathophysiology of delirium: A focus on the role of aberrant stress responses. Journal of Psychosomatic Research. 2008;65:229-238.

44. Davis DH, Muniz-Terrera G, Keage HA, Stephan BC, Fleming J, Ince PG, Matthews FE, Cunningham C, Ely EW, MacLullich AM, Brayne C. Association of Delirium With Cognitive Decline in Late Life: A Neuropathologic Study of 3 Population-Based Cohort Studies. JAMA psychiatry. 2017;74:244-251.

45. Evered L, Silbert B, Scott DA, Zetterberg H, Blennow K. Association of Changes in Plasma Neurofilament Light and Tau Levels With Anesthesia and Surgery: Results From the CAPACITY and ARCADIAN Studies. JAMA neurology. 2018.

46. Xie Z, Xu Z. General anesthetics and beta-amyloid protein. Progress in neuropsychopharmacology \& biological psychiatry. 2013;47:140-146.

47. Shahim P, Tegner Y, Marklund N, Blennow K, Zetterberg H. Neurofilament light and tau as blood biomarkers for sports-related concussion. Neurology. 2018. 
48. Neselius S, Zetterberg H, Blennow K, Marcusson J, Brisby H. Increased CSF levels of phosphorylated neurofilament heavy protein following bout in amateur boxers. PloS one. 2013;8:e81249.

49. Cunningham C, Maclullich AM. At the extreme end of the psychoneuroimmunological spectrum: delirium as a maladaptive sickness behaviour response. Brain, behavior, and immunity. 2013;28:1-13.

50. Oh ES, Fong TG, Hshieh TT, Inouye SK. Delirium in Older Persons: Advances in Diagnosis and Treatment. Jama. 2017;318:1161-1174.

51. Olsson B, Lautner R, Andreasson U, Ohrfelt A, Portelius E, Bjerke M, Holtta M, Rosen C, Olsson C, Strobel G, Wu E, Dakin K, Petzold M, Blennow K, Zetterberg H. CSF and blood biomarkers for the diagnosis of Alzheimer's disease: a systematic review and meta-analysis. The Lancet Neurology. 2016;15:673-684.

52. Vagberg $M$, Norgren $N$, Dring A, Lindqvist $T$, Birgander $R$, Zetterberg $H$, Svenningsson $A$. Levels and Age Dependency of Neurofilament Light and Glial Fibrillary Acidic Protein in Healthy Individuals and Their Relation to the Brain Parenchymal Fraction. PloS one. 2015;10:e0135886. 


\section{FIGURE LEGEND}

\section{Figure 1. Flow chart in the hip fracture population.}

Figure 2. Neurofilament light (NFL) concentrations according to delirium status in hip fracture patients. A. NFL in serum pre- and postoperatively. One preoperative outlier value (no delirium $y=545$ ) and eight postoperative outlier values (no delirium $y 1=862, y 2=729, y 3=640$ and delirium $y 4=930, y 5=848$, $y 6=672, y 7=597, y 8=590$ ) were removed for visual purposes but not from the statistical analysis. B. NFL in cerebrospinal fluid. The black and grey lines represent the median. The p values are from the Mann Whitney- $U$ test.

Figure 3. Neurofilament light (NFL) concentrations in delirium subgroups. A. NFL in serum preoperatively in delirium subgroups. Three outliers were excluded from the figure $y l=1211$ and $y 2=805$ (preoperative delirium) and $y 3=545$ (no delirium) for visual purposes but not from the statistical analysis. B. NFL in serum postoperatively in delirium subgroups. Three outliers were excluded from the figure $y l=930$ and $y 2=848$ (preoperative delirium) and $y 3=862$ (subsyndromal delirium) for visual purposes but not from the statistical analysis. C. Changes of serum NFL pre- to postoperatively in delirium subgroups. Six outliers had values above the upper margin of this figure: $y 1=457, y 2=304$ and $y 3=305$ (incident delirium), $y 4=591$ (preoperative), $y 5=603$ and $y 6=282$ (subsyndromal), and one outlier was below the lower margin, $y 4=-249$ (no delirium). D. NFL in cerebrospinal fluid in delirium subgroups. Three outliers were removed for visual purposes $(y l=10016, y 2=10020, y 3=10027)$. The grey line represents the median. All measurements are in the full hip fracture group. The p-values are from the Kruskal-Wallis (A-B) and Mann Whitney- $U$ tests (CD).

Figure 4. Change in serum NFL according to delirium status with exclusion of preoperative delirium. Change was measured in all hip fracture patients and after stratifying upon dementia status. The black line represents the median. The p-values are from the Mann Whitney- $U$ test. 
Figure 5. Correlation between cerebrospinal and serum concentrations of neurofilament light. 
Table 1. Demographics and levels of Neurofilament Light (NFL)

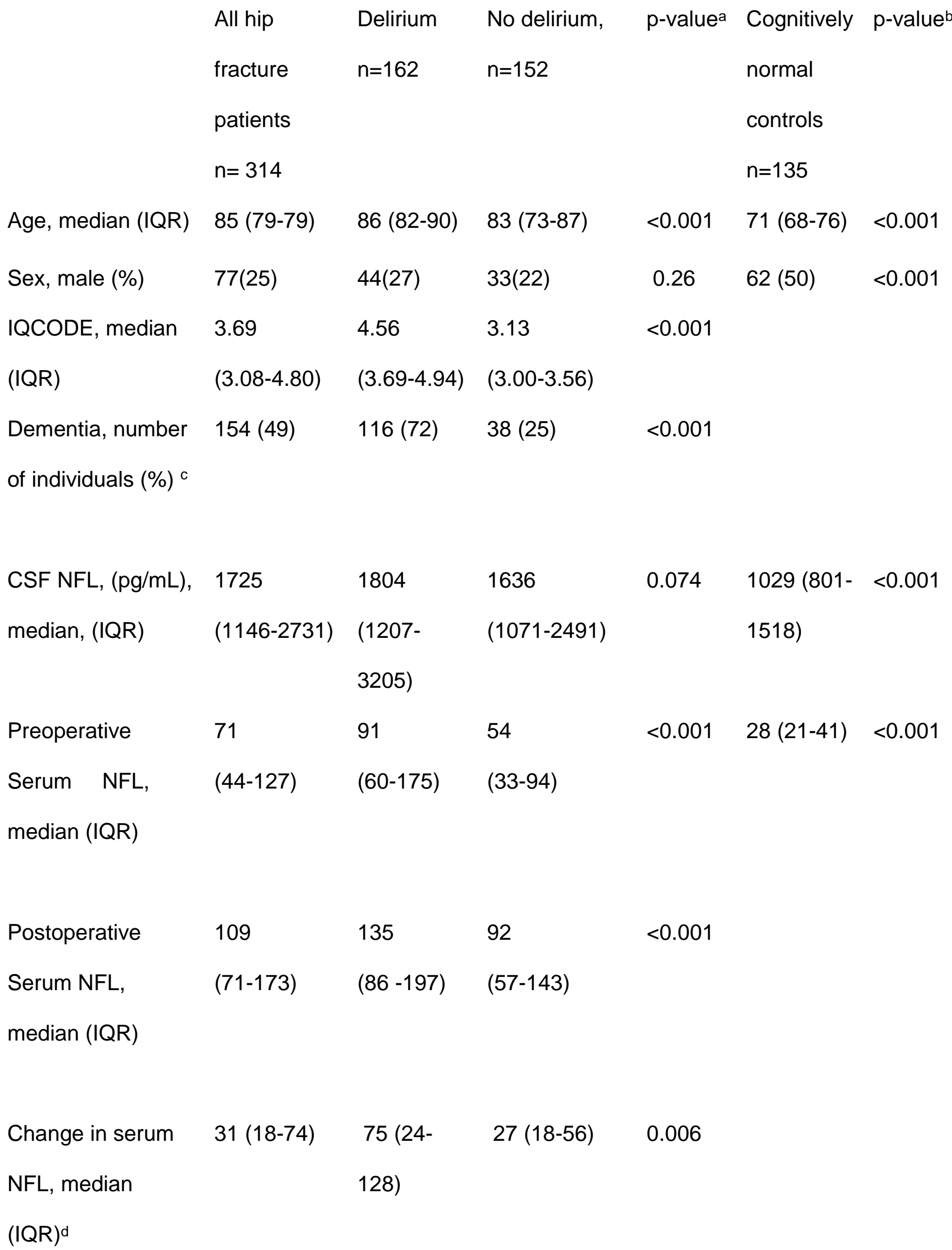


IQR- InterQuartile Ratio. IQCODE- Informant Questionnaire of Cognitive Decline. CSF -

Cerebrospinal fluid. NFL- NeuroFilament Light

a Hip fracture patients with delirium versus without delirium

b Cognitively normal controls versus all hip fracture patients

${ }^{\mathrm{C}}$ Consensus in an expert panel

d Postoperative NFL- preoperative NFL (preoperative delirium excluded) 
Table 2. Neurofilament light in serum and CSF in hip fracture patients in delirium after dementia stratification

Dementia

No delirium Delirium

p-value No delirium

Delirium

p-

value

Cerebrospinal $1561-2093$

$1848(1191-$

0.98

$1259(881-$

2091)

$1736(1182-$

0.14

fluid NFL $\quad(n=2)$

3533)

$97(60-182)$

0.33

$42(23-73)$

$95(61-168)$

serum NFL

Postoperative 119 (79-156)

$133(87-204)$

0.18

$80(43-126)$

$136(75-194)$

serum NFL

Change in $23(10-48)$

$94(41-157) \quad<0.001 \quad 28(19-58)$

$46(-0.6-99)$

serum NFLa

${ }^{a}$ Change is reported excluding patients with preoperative delirium 
Supplementary table 1. Regression analyses of NFL concentrations (dependent variable) with delirium and age as independent variables. NFL values were transformed with the natural logarithm in order to approximate distribution of residuals to the normal distribution.

$\begin{array}{llll}\text { Ln (Preoperative } & \text { Ln(Postoperative } & \text { Ln (Preoperative } & \text { Ln (Postoperative } \\ \text { serum NFL) all hip } & \text { serum NFL) all hip } & \text { serum NFL) in the } & \text { serum NFL) in the } \\ \text { fracture patients } & \text { fracture patients }^{\text {a }} & \text { dementia-free } & \text { dementia-free } \\ & & \text { stratum }^{\text {a }} & \text { stratum }^{\text {a }}\end{array}$

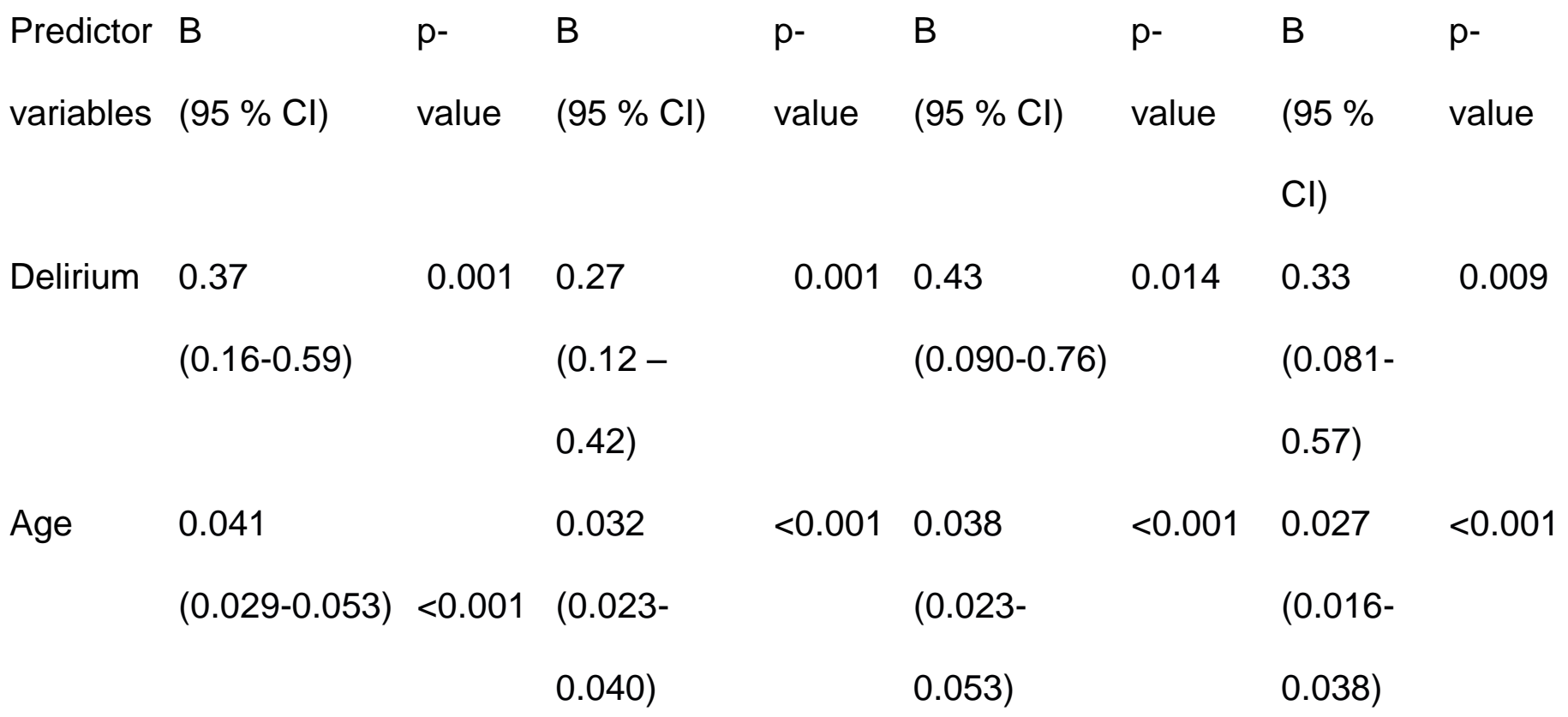


$\mathrm{Ln}($ Change in NFL) in hip $\mathrm{Ln}($ Change in NFL) in patients

fracture patients

(preoperative delirium

excluded) ${ }^{\mathrm{a}}$

with dementia (preoperative

delirium excluded) ${ }^{\mathrm{a}}$
Ln(Cerebrospinal fluid NFL) in hip fracture patients (subsyndromal delirium excluded) ${ }^{a}$

Predictor $\mathrm{B}(95 \% \mathrm{Cl}) \quad \mathrm{p}-\quad \mathrm{B}(95 \% \mathrm{Cl}) \quad \mathrm{p}$-value $\quad \mathrm{B}(95 \% \mathrm{Cl}) \quad$ pvariables value

Delirium $\quad 0.57(0.093-1.05) \quad 0.020 \quad 0.93(0.26-1.6)$

$0.009 \quad 0.21(-0.087-$

0.16

\begin{tabular}{|c|c|c|c|c|c|}
\hline Age & 0.027 (0.005- & 0.017 & $0.004(-0.046-$ & 0.88 & 0.023 (0.005- \\
\hline & $0.050)$ & & $0.054)$ & & $0.40)$ \\
\hline
\end{tabular}

B $(95 \% \mathrm{Cl})$ - Regression coefficient B with $95 \%$ confidence intervall

a dependent variable 

